\title{
MedienPädagogik
}

Zeitschrift für Theorie und Praxis der Medienbildung

\section{Editorial: Informationstechnische Bildung und Medienerziehung}

Annemarie Hauf-Tulodziecki

Die schulische Medienerziehung bzw. die Medienbildung hat ihre derzeit hohe öffentliche Aufmerksamkeit nicht zuletzt der Tatsache zu verdanken, dass gerade die so genannten «Neuen Medien» - heute oft mit den Schlagworten «Multimedia» oder «Internet» abgekürzt - wichtige Erziehungs- und Bildungsmassnahmen erforderlich machen.

In der öffentlichen Diskussion gehören inhaltliche Kurzschlüsse zur Tagesordnung: Wenn von «Medienkompetenz» die Rede ist, verbirgt sich dahinter häufig nur ein «Internetführerschein». Diese Beobachtung ärgert nicht nur Medienpädagogen, sondern auch Vertreter einer Informationstechnischen Bildung ${ }^{1}$, da berechtigte pädagogische Ansprüche beider Gruppen wenig Beachtung finden. Mittlerweile gibt es eine Reihe von Empfehlungen von BLK und KMK zu diesem Thema, in denen zudem deutlich auf die wechselseitigen Bezüge hingewiesen werden.

Die wachsende Bedeutung des Mediums Computer und die weiter zunehmende Digitalisierung traditioneller Medien erfordert sowohl für die Medienerziehung als auch für die Informatische Bildung eine Weiterentwicklung ihrer Ziele und Inhalte. Die Gesellschaft für Informatik e.V. hat 1999 in ihrer Empfehlung «Informatische Bildung und Medienerziehung» aus ihrer Perspektive dargestellt, welche Aufgaben auf die Medienerziehung zukommen, wenn auch die Informations- und Kommunikationstechnologien bzw. die computerbasierten Medien in einer angemessenen Form thematisiert werden sollen.

Erste praktische Ansätze, Inhalte aus beiden Bereichen bewusst zusammenzuführen, auch unter einer gemeinsamen Fach- oder Kursbezeichnung, sind häufig additiv und orientieren sich - trotz vorhandener Überschneidungen - an den etablierten medienpädagogischen oder an den informatischen Unterrichtsprinzipien.

In dem vorliegenden Themenheft wird aus einer übergeordneten, medienpädagogischen Perspektive die Frage gestellt, wie eine konsistente Weiterentwicklung der Medienerziehung unter angemessener Berücksichtigung der informatischen Perspektive aussehen kann. Dieser Frage gehen die vorliegenden Beiträge aus verschiedenen Blickwinkeln nach. Die Beiträge von Herzig, Schulte und Wagner zeigen einen systematischen Zugang:

1 Die Tatsache, dass das Thema noch in der Diskussion (nicht konsolidiert?) ist, zeigt sich u.a. auch darin, dass in den verschiedenen Beiträgen die Bezeichnungen informationstechnische, informationstechnologische und informatische Bildung sowie Medienerziehung und Medienbildung nebeneinander verwendet werden. Wir haben bewusst von einer Vereinheitlichung abgesehen, da sie zum jetzigen Zeitpunkt auch die jeweilige Herkunft der Ansätze illustrieren. 
Bardo Herzig entwickelt mit einem zeichenorientierten Ansatz einen interdisziplinären Zugang zu einem gemeinsamen Medienbegriff. Über die Semiotik wird eine theoretische Grundlage geschaffen, die es ermöglicht, medienpädagogische und informationstechnische Sichtweisen im Bereich der Informations- und Kommunikationstechnologien miteinander zu verbinden.

Carsten Schulte untersucht Sichtweisen der Fachwissenschaft und Fachdidaktik Informatik, die medialen Aspekte des Computers berühren, entwickelt hieraus Aufgabenbereiche einer «informatischen Medienbildung» und weist nach, dass mit innen die Anforderungen der «medienpädagogischen Aufgabenbereiche» bezogen auf computerbasierte Medien erfüllt werden können.

Aus einer eher medienpädagogischen Perspektive beschreibt Wolf-Rüdiger Wagner Anforderungen, durch aus spezifischen Gefahren durch die Informationstechnologien erwachsen: Medienkompetenz als Fähigkeit zum Selbstschutz erfordert (Un-) Sicherheitsbewusstsein, das Recht auf informationelle Selbstbestimmung, ein technisches Grundverständnis sowie ein angemessenes Kommunikationsverhalten. $\mathrm{Zu}$ vergleichbaren Ergebnissen kommt übrigens auch Klaus Brunnstein in seinem Vortrag bei der Fachtagung «Informatikunterricht und Medienbildung», die im September 2001 stattgefunden hat. Er plädierte für eine drastische Revision der Informatikcurricula: Statt vertiefender Programmierkenntnisse sei die Beherrschbarkeit unsicherer Informationstechniken in den Vordergrund zu stellen. Verantwortlicher Umgang mit diesen Techniken bedeutet die kontrollierte Nutzung, Verständnis der Leistungsfähigkeit und Grenzen von Produkten sowie Kenntnisse der wirtschaftlichen und rechtlichen Rahmenbedingungen ${ }^{2}$.

Die weiteren Beiträge sind eher als eine pragmatische Annäherung an das Thema zu verstehen.

Michael Weigend stellt konkrete Unterrichtsvorschläge vor. Seine medienbezogenen Projektthemen, die eine Kombination der Fächer Informatik und Erziehungswissenschaft voraussetzen, enthalten interessante Anregungen. Die anstehende praktische Erprobung wird Auskunft geben über den pädagogischen Ertrag im Hinblick auf die verschiedenen fachlichen und überfachlichen Zielbereiche, auf Durchführbarkeit und Akzeptanz bei Lernenden und Lehrenden.

In ihrer Studie zeigt Irene Langner, wie sich - als Folge der eingangs genannten bundesweiten Empfehlungen - in einigen Bundesländern bei dem Thema Internet allmählich eine Annäherung zwischen den Ansätzen von Informations- und Kommunikationstechnologischer Grundbildung, Medienerziehung und Informatik vollzieht und vergleicht diese Entwicklung mit dem in Japan vorliegenden informationsorientierten Konzept.

2 Vgl. Brunnstein, K.: Mit IT-Risiken umgehen lernen: über Probleme der Beherrschbarkeit komplexer Informatiksysteme. In: Keil-Slawik, R.; Magenheim, J. (Hrsg.): Informatikunterricht und Medienbildung. INFOS 2001. 9. GI-Fachtagung Informatik und Schule 17.-20. September 2001 in Paderborn. GI: Bonn 2001. 
Sollen neue Ziele und Inhalte umgesetzt werden, ist eine Verankerung in der Lehrerausbildung ein wichtiger Schritt. Ein Ansatz hierzu ist das von Olaf Kos und Dieter Schaale vorgestellte Mindestcurriculum zur Vermittlung von Informatischer Bildung für Lehramtsstudierende. Die Autoren verweisen auch auf umfassendere Studienangebote anderer Universitäten, die als Zusatzqualifikationen ausgewiesen sind und in denen Veranstaltungen aus dem Bereich der Erziehungswissenschaft, der Fachdidaktiken zum Teil mit einem Schwerpunkt bei der Didaktik der Informatik angeboten werden, sowie auf das Hochschulnetzwerk «Lehrerausbildung und neue Medien» (http://www.lehrerbildung-medien.de).

In diesem Zusammenhang sind auch unterstützende und begleitende Massnahmen zu erwähnen, um Hochschulen, Studienseminare zu motivieren, entsprechende Angebote zu diesen Themen bereit zu stellen und diese Angebote für Abnehmer/innen attraktiv zu machen. Ein Beispiel ist das «Portfolio: Medien.Lehrerbildung», mit dem Aus- und Fortbildungsaktivitäten im Bereich «medienpädagogischer Kompetenz» dokumentiert, Arbeitsergebnisse gesammelt und präsentiert werden können.

Insgesamt soll das Themenheft einen Einblick in die aktuellen Diskussionen zum Thema und die vorliegenden (Zwischen-)Ergebnisse bieten. Interessant wird es sein, die einzelnen Beiträge auch nach der Frage zu lesen, inwieweit die einzelnen - theoretischen und praktischen - Ansätze miteinander «kompatibel» sind. Es lohnt sich, die Entwicklung weiter zu verfolgen. 